\title{
O USO DE BANCOS DE DADOS LOCAIS EM UM AMBIENTE COLABORATIVO DE APRENDIZAGEM BASEADO NA WEB
}

\author{
Fábio de Jesus Lima Gomes* \\ José Valdeni de Lima**
}

\begin{abstract}
Resumo
Este artigo descreve um trabalho para promover o aprendizado em um ambiente colaborativo de aprendizagem baseado na web. Tal trabalho consiste na possibilidade de que cada estudante participante do ambiente possua seu próprio banco de dados local, que servirá como repositório para suas pesquisas no âmbito do assunto que o mesmo encontra-se em estudo. Cada banco de dados local será disponibilizado para consulta aos demais estudantes participantes do ambiente, assim, diversos bancos de dados locais podem ser vistos pelos estudantes como um único banco de dados, proporcionando a troca de informações entre os estudantes e a melhoria do processo de aprendizagem.
\end{abstract}

Palavras-chave: integração de dados, banco de dados, educação a distância, aprendizagem colaborativa.

\section{THE USE OF LOCAL DATABASES IN A COLLABORATIVE LEARNING ENVIRONMENT WEB-BASED}

\begin{abstract}
The present paper describes a work of a collaborative learning environment webbased. It aims at promoting for each student a data base which will be used as a repository of his surveys for the subjects he is dealing with. Each of these student database will be available for the other students in the group. This way several databases can be visualized as they were only one for every student. It will make the information exchange among students easier. It shall work for the learning improvement as well.
\end{abstract}

Keywords: data integration, database, distance learning, collaborative learning.

\section{Introdução}

Segundo Valente (1997), a real função do computador na área de ensino é provocar mudanças na abordagem pedagógica vigente ao invés de colaborar com o professor para tornar mais eficiente o processo de transmissão do conhecimento. Tal função pode ser conseguida através de um software que possa auxiliar os estudantes no processo de aprendizagem, o software educacional.

\footnotetext{
Doutorando PPGIE / UFRGS

*** Professor PPGIE / UFRGS

V. $2 \mathrm{~N}^{\circ} 2$, Novembro, 2004
} 
O software educacional também possui o objetivo de auxiliar o professor no processo de ensino, fazendo com que o mesmo tenha a seu dispor valiosos recursos para ajudá-lo nas tarefas didáticas junto aos seus alunos. Uma das áreas de pesquisa em software educacional é a CSCL (Computer Supported Collaborative Learning Aprendizagem Colaborativa Assistida por Computador) (CSCL 2004), que se constitui da utilização de computadores em rede e de um aplicativo apropriadamente escolhido ou projetado dentro de um contexto de instrução que apóie os processos de aprendizado em grupo e geralmente é utilizado para educação a distância.

A educação a distância nasceu sob o signo da democratização do saber. Trata-se de uma inovação educativa que tem por objetivo maior gerar condições de acesso à educação para todos aqueles que, por um motivo ou outro, não estejam sendo atendidos satisfatoriamente pelos meios tradicionais de ensino. É uma inovação educativa, mas já tem muitos anos de experiência (Todorov, 1994).

Dessa forma, a educação a distância surge como opção para atender a demanda de educação continuada. Também, em sintonia com os desenvolvimentos tecnológicos, esta modalidade de ensino apresenta sua evolução ao longo do tempo, usando destes mesmos meios tecnológicos para melhorar a qualidade de seus programas. Dentre os diversos meios tecnológicos que podem ser utilizados para educação a distância, merece destaque a Internet.

Porter (1997) afirma que a Internet e a World Wide Web (WWW) são, sem dúvida, o maior fenômeno desta última década na área da informática e das comunicações. É um daqueles inventos que, por serem tão revolucionários, passam imediatamente a fazer parte do dia-a-dia das pessoas, e não se consegue imaginar mais o mundo sem ele. Foi assim, com a imprensa, o rádio, o telefone, a televisão, e agora ocorre de novo com a Internet.

Segundo Gomes (2000), a Internet tem um grande potencial educacional, pois, além das facilidades de comunicação, consegue agregar diferentes mídias (texto, imagem, áudio, até as mais variadas simulações), criando ricos espaços de aprendizagem. Dessa forma, a Internet surge como uma alternativa valiosa para a educação a distância, uma vez que proporciona alto grau de interação e a WWW é apontada como a mais poderosa ferramenta da Internet para fins educacionais.

A WWW se apresenta como uma tecnologia capaz de atender às expectativas dos pesquisadores da área de ensino e aprendizagem a distância, proporcionando soluções para o problema do oferecimento de educação e treinamento em larga escala, a custos mais acessíveis que os atuais, permitindo a publicação de material didático, aplicação de tutoriais, aplicação de provas e testes, comunicação com os estudantes e apresentação de aulas a distância (conferência multimídia). Portanto, com o advento da Internet e principalmente da WWW, começaram a ser desenvolvidos ambientes virtuais para educação a distância, tais como, e-Proinfo, AulaNet, WebCT, TelEduc (2004).

Estes ambientes possuem ferramentas de suporte a educação a distância, tais como, fórum de discussão, salas de bate-papo e áreas de armazenamento de dados para alunos e professores. Esta área de armazenamento constitui um espaço no computador que funciona como servidor do ambiente, onde se pode inserir, alterar, excluir e disponibilizar dados, que geralmente estão na forma de arquivos ou de links. Como exemplo desta área de armazenamento, citamos o Portfólio do ambiente TelEduc. Tal área tem um espaço limitado para armazenamento, assim, o aluno não consegue 
armazenar todas as informações que gostaria que fossem armazenadas e disponibilizadas para os demais estudantes.

Este artigo mostra uma outra maneira de proporcionar uma área de armazenamento de dados aos usuários de um ambiente de ensino. Tal maneira permite que cada usuário tenha armazenado localmente seu próprio banco de dados, que é similar à base do domínio disponibilizada pelo ambiente, sendo que este banco de dados local é constituído pelas pesquisas do estudante. Quando o estudante está alimentando seu banco de dados local com suas pesquisas, informações adicionais são armazenadas para fins de recuperação. Isto permite que qualquer usuário do sistema visualize, através do browser, as informações disponíveis de seu grupo de estudos, através de consultas aos bancos de dados locais dos estudantes.

Na seção 2, é apresentada a aprendizagem colaborativa. A implementação do trabalho deste artigo está na seção 3 e na seção 4, são mostradas as conclusões.

\section{Aprendizagem Colaborativa}

$\mathrm{Na}$ área educacional, é fundamental que se encontrem recursos, se possível integrados em ambientes mais completos, que apóiem a aprendizagem colaborativa, permitindo que, mesmo a distância, os aprendizes possam interagir, construir conhecimento e compartilhar suas construções.

Quando um trabalho é feito de maneira colaborativa, o resultado aparenta ser bem melhor que quando é obtido de uma abordagem individual. Desenvolvendo atividades colaborativas, os estudantes trocam experiências e têm contato com outras possibilidades para resolver o mesmo problema.

Segundo Hiltz e Turoff (1993), a aprendizagem colaborativa pode ser definida como um processo que exige a participação ativa e a interação, tanto da parte dos estudantes quanto dos professores. Nesta perspectiva, o conhecimento é visto como uma construção social e o ambiente em que isto ocorre deve proporcionar a interação entre os participantes, a avaliação e a colaboração.

O aprendizado colaborativo é uma atividade social que envolve alunos compartilhando conhecimentos e adquirindo outros novos, processo que tem se nomeado como construção social do conhecimento e está vinculado à teoria do construtivismo social que assume que o aprendizado se dá entre pessoas e não entre pessoas e coisas e se fundamenta na idéia de que todos juntos somos mais inteligentes que cada um por si só.

No esquema do aprendizado colaborativo o estudante deixa de ser um agente passivo que recebe a informação do professor e passa a ser um agente ativo na construção do conhecimento.

Tinto, Goodsell \& Russo (1995) apontam que o aprendizado colaborativo promove a discussão interativa entre os estudantes e o professor, favorecendo o desenvolvimento do pensamento crítico. Os estudantes se sentem dentro dos grupos de discussão mais à vontade para expressar seus pensamentos. $\mathrm{O}$ trabalho em equipe estimula uma interação verbal maior. Neste tipo de aprendizado, os estudantes se envolvem mais tanto social quanto academicamente e reconhecem que a qualidade do aprendizado é melhor que com os métodos tradicionais porque eles têm realmente que aprender e não apenas memorizar. 
Para Harasin (1989), a aprendizagem ativa tem sido uma das importantes contribuições dos ambiente on-line, porque os alunos podem se comunicar pelas diferentes formas de comunicação entre professores e alunos, síncrona e assíncrona, conectando-se regularmente.

O que se pretende neste artigo é que o estudante tenha uma participação ativa no seu processo de aprendizagem, através das pesquisas em diversos meios (WWW, livros, revistas etc.) para o posterior armazenamento em seu banco de dados local, como também, espera-se que o estudante tenha atividades colaborativas, através da disponibilização do seu banco de dados para consulta dos outros participantes do ambiente de ensino.

\section{O uso de bancos de dados locais em um ambiente colaborativo de aprendizagem baseado na web}

Um ambiente de ensino geralmente possui uma base do domínio que armazena o conteúdo instrucional que vai ser apresentado ao aluno: lições, exercícios, informações complementares, ajuda, dentre outros.

$\mathrm{Na}$ arquitetura a ser apresentada, além da base do domínio, cada estudante tem seu próprio banco de dados local, chamado de cooperante, e constituído por suas pesquisas. A utilização de bancos de dados cooperantes nos traz algumas vantagens:

- Compartilhamento de dados - se os bancos de dados locais cooperantes estão conectados, qualquer usuário habilitado pode ser capaz de fazer acesso a dados disponíveis nestes bancos de dados;

- Confiabilidade e disponibilidade - se um banco de dados não estiver conectado ou falhar no sistema de integração, os bancos de dados remanescentes podem ser capazes de continuar operando. Assim, a falha de um banco de dados local cooperante não implica necessariamente no desligamento do sistema;

- Aceleração de processamento de consultas - se uma consulta envolve dados em diversos bancos de dados, é possível dividi-la em sub-consultas que podem ser executadas em paralelo;

- Independência de localização - se os dados estiverem localizados em vários bancos de dados, isso deve ser imperceptível a qualquer cliente;

- $\quad$ Escalabilidade - como em cada banco de dados local cooperante ocorre inclusão de dados (pesquisas do estudante), a base do domínio pode sofrer considerável aumento de conteúdo (informação);

- Autonomia e Descentralização - os bancos de dados que fornecem os dados para o sistema de integração são autônomos, ou seja, suportam aplicações locais, podendo sofrer alterações nos dados, como também, ocorrendo falha no servidor do ambiente, não há prejuízo no processo de ensino e aprendizagem;

Esse banco de dados local cooperante pode ser compartilhado com o grupo, permitindo que os participantes tenham acesso às pesquisas individuais dos outros participantes, proporcionando uma maior integração e troca de informações e possibilitando a construção e o acúmulo de informação através das bases do domínio do próprio ambiente, como também, através dos bancos de dados individuais de cada aluno, contribuindo assim para um ambiente efetivamente colaborativo. $\mathrm{O}$ compartilhamento desse banco de dados é de fundamental importância para a melhoria 
no processo de aprendizagem. Dessa forma, a integração dos bancos de dados locais cooperantes dos estudantes se faz necessária e se torna uma opção para o aprendizado colaborativo. Um dos objetivos deste trabalho é efetuar a integração dos bancos de dados locais cooperantes e é mostrada nas seções seguintes.

\subsection{Integração de banco de dados}

A disponibilização e integração de dados surgem como tendência de serviços oferecidos pela WWW. O objetivo de um sistema de integração de dados é oferecer aos usuários uma interface uniforme de acesso a diferentes fontes de dados, de forma que os usuários definam consultas especificando o que desejam saber e o sistema determine onde a informação pode ser encontrada e, em seguida, apresente as respostas para as consultas do usuário. Um sistema de integração de dados deve possuir características de distribuição, autonomia e integração através dos conceitos de bancos de dados federados (Busse et al, 1999), que consiste na integração de vários bancos de dados cooperantes e autônomos, mas que participam de uma federação possibilitando o compartilhamento parcial e controlado dos seus dados.

Tal integração deve prover as transparências desejadas de bancos de dados distribuídos (Ö̈zsu, 2001), principalmente, a transparência de localização, que consiste no acesso a um recurso remoto, neste caso, um banco de dados, sem precisar informar o seu endereço e sem conhecer a sua localização física. Os usuários precisam ter uma visão única do sistema. Entretanto, cada banco de dados local cooperante precisa fornecer, através de cada esquema interno, a porção de dados que está disponível e visível para os usuários globais. É através da integração de esquemas que se obtém o mapeamento entre a visão dos usuários globais e os esquemas locais.

Segundo Thomas (1994), a integração de esquemas é o processo de desenvolvimento de um esquema conceitual, livre de duplicações e heterogeneidades, que integra uma coleção de esquemas locais. Em outras palavras, em um sistema de bancos de dados cooperantes, as informações globais devem ser traduzidas de modo a solucionar os conflitos que podem ocorrer durante a integração de esquemas.

Em um sistema de integração de dados, o processamento de consultas globais é a capacidade de combinar os dados de diferentes sistemas de banco de dados em uma única operação de consulta (Thomas, 1994). O problema principal de consultas globais está em transformar uma consulta em uma linguagem de alto nível especificada pelo usuário em sub-consultas equivalentes, mantendo a corretude na hora da tradução da consulta.

A arquitetura para a integração e utilização dos bancos de dados locais cooperantes é mostrada na próxima seção.

\subsection{Arquitetura}

A arquitetura implementada é uma instância da arquitetura cliente/servidor em três camadas, onde na primeira camada é descrita a interface com o usuário, a segunda camada é representada pelos objetos distribuídos, onde está contido a lógica do negócio e na última camada são apresentados os diversos bancos de dados a serem integrados, podendo ser visualizada na figura 1 e é composta dos seguintes componentes:

- $\quad$ interface do usuário escrita em Java (2004) e HTML (2004); 
- módulo servidor escrito em Java/Servlets (2004) para acessar o banco de dados armazenado no computador que funciona como servidor do ambiente e para realizar o processamento das consultas aos bancos de dados locais cooperantes;

- módulos servidores escritos em Java/RMI (2004) para acessar os bancos de dados locais cooperantes, que são vistos pelo ambiente como objetos distribuídos.

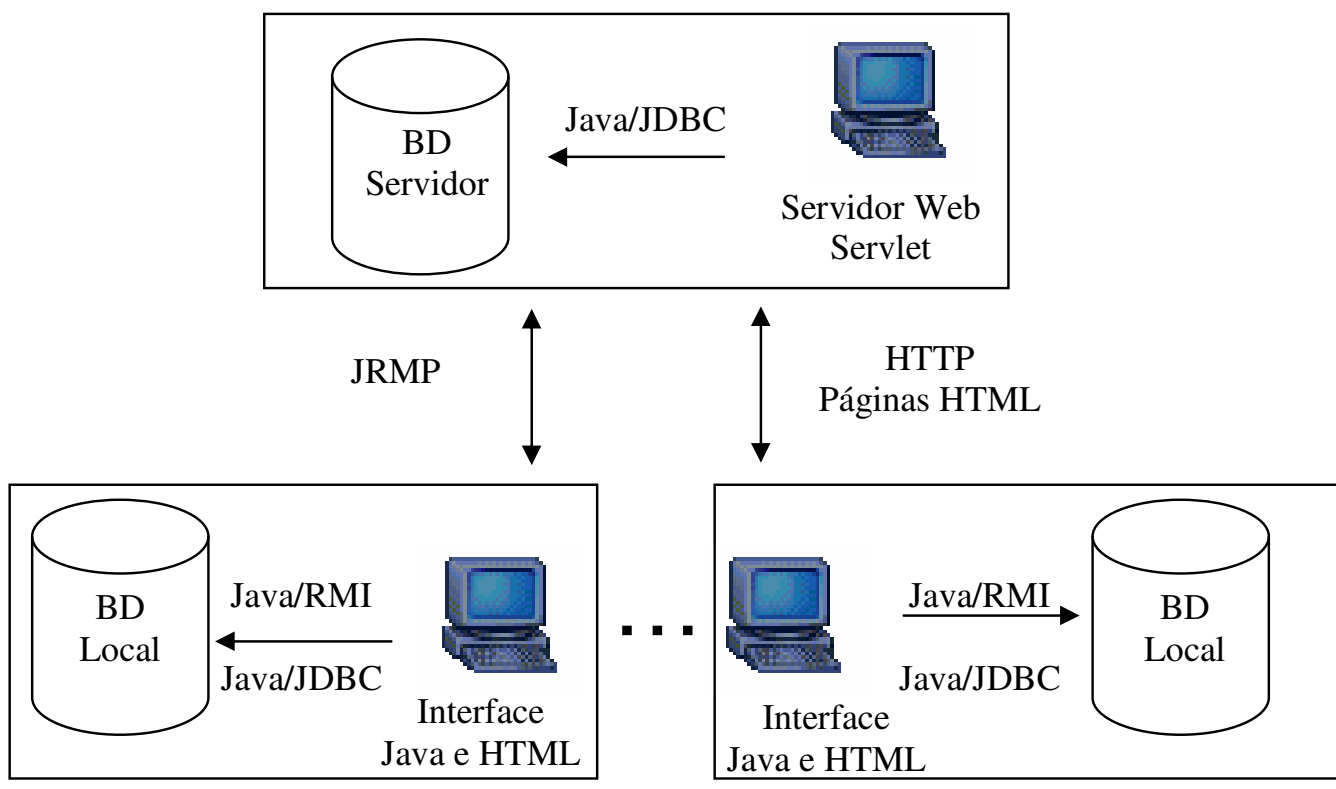

Usuários

Figura 1: Arquitetura proposta para o uso de bancos de dados locais em um ambiente colaborativo de aprendizagem baseado na web

A interface é constituída por dois módulos: (1) uma página HTML que é visualizada pelo browser para utilização do ambiente, incluindo uma interface de consulta aos bancos de dados locais cooperantes dos demais estudantes e (2) uma aplicação local que possibilita ao estudante armazenar suas pesquisas no banco de dados local, bem como, permitir a disponibilização do seu banco de dados local.

Esta aplicação local é disponibilizada ao usuário após o seu cadastro e tem estrutura visual e funcionalidades semelhantes à do ambiente, dando uma visão de que a aplicação local é uma extensão do ambiente. Assim como, possibilita ao estudante armazenar no banco de dados local suas pesquisas feitas na WWW, em livros, revistas, periódicos etc.

Outra função da aplicação local, é permitir ao usuário escolher em disponibilizar ou não o seu banco de dados local cooperante. Caso queira disponibilizá-lo, a aplicação irá executar localmente uma classe Java que ativa um servidor RMI, responsável por disponibilizar o banco de dados local como um objeto remoto, para a consulta de outros usuários. Após executar a classe, irá aparecer uma janela informando que o servidor RMI está ativo, com um botão que é utilizado para parar o servidor RMI, caso o usuário não mais queira a disponibilização do seu banco de dados, conforme a figura 2 . 


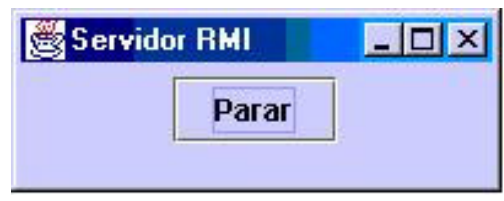

Figura 2: Janela ativa após execução da classe que disponibiliza o BD local

Quando um usuário solicita uma consulta aos bancos de dados locais cooperantes, existe um servlet no servidor web responsável por localizar o servidor RMI e o objeto remoto, neste caso, o banco de dados local cooperante de cada usuário. Após a localização, o servlet cria uma instância do objeto remoto e se torna um cliente RMI do servidor RMI localizado. Para executar a consulta, o servlet faz a chamada de um método remoto, através do protocolo JRMP (Java Remote Method Protocol) (RMI, 2004), que retorna o resultado da consulta em cada banco de dados local cooperante. Logo após, o servlet gera uma página HTML com os resultados das consultas e exibe ao usuário que solicitou a consulta.

Nesta arquitetura, a API RMI está sendo utilizada para comunicação entre objetos distribuídos (remotos) e, juntamente com os drivers JDBC (2004) para acesso aos bancos de dados locais cooperantes. A estratégia principal é manter vários servidores RMI rodando, permitindo com isso, a possibilidade de rodar solicitações de consultas aos bancos de dados locais cooperantes em paralelo. Isso somente é possível pela característica multi-threading da linguagem Java.

O acesso ao conteúdo instrucional do ambiente é feito através do protocolo HTTP com chamadas ao servlets, e recebendo como respostas páginas HTML. Os servlets são responsáveis pela conexão com o banco de dados do servidor através de um driver JDBC.

A API JDBC está sendo utilizada para acessar todas as bases de dados, quer a base de dados localizada no servidor, quer os bancos de dados locais cooperantes. A funcionalidade básica dessa API é prover basicamente: conexão com bancos de dados, enviar comandos SQL e processar resultados.

Para o processamento das consultas, um servlet constrói as sub-consultas de acordo com o banco de dados local, visto que o esquema do banco de dados local é semelhante ao banco de dados do servidor do ambiente que armazena a base do domínio do curso a ser ensinado e, caso o usuário possua um SGBD, este é informado quando o usuário se cadastra no ambiente. Dessa forma, as sub-consultas SQL são construídas pelo servlet de maneira correta e sem a necessidade de se resolver conflitos, que porventura venham a ocorrer no procedimento de integração de dados.

O tratamento de heterogeneidades pode ser totalmente implementado nessa arquitetura. As diferenças de hardware, software e sistemas operacionais foram totalmente suportadas pelas próprias características da linguagem Java. A API JDBC também facilita muito o tratamento das heterogeneidades entre os Sistemas de Gerenciamento de Banco de Dados (SGBDs), pois fornece uma interface única dos bancos de dados cooperantes e esconde as características individuais e as diferenças dos dialetos SQL.

$\mathrm{Na}$ seção seguinte são apresentadas as principais páginas do protótipo e é explicado resumidamente a sua utilização. 


\subsection{Utilização do Protótipo}

Após a entrada no ambiente, o usuário está habilitado a consultar os bancos de dados locais cooperantes. O sistema irá apresentar a página mostrada na Figura 3, que permite ao usuário escolher o curso cadastrado que se deseja consultar.

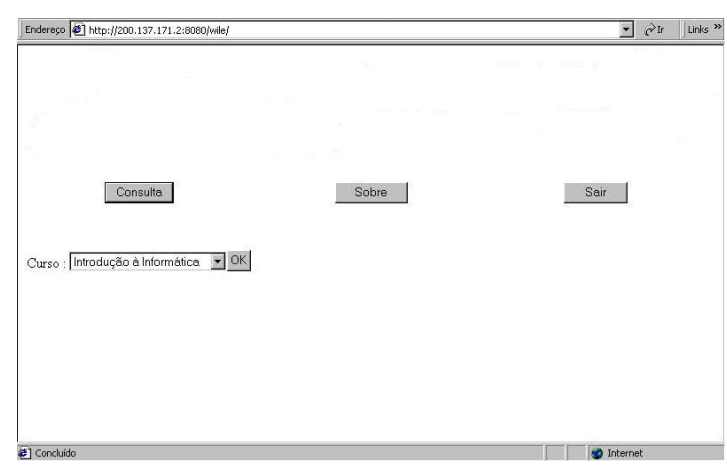

Figura 3: Consulta cursos cadastrados

Após escolher o curso, será exibida uma página com as lições referentes ao curso escolhido, conforme a figura 4.

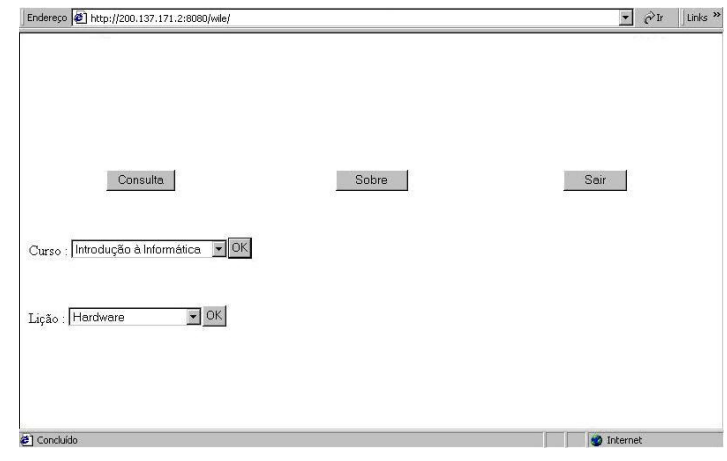

Figura 4: Consulta lições do curso

Após a escolha da lição, será exibida uma página com os tópicos referentes à lição escolhida, de acordo com a figura 5.

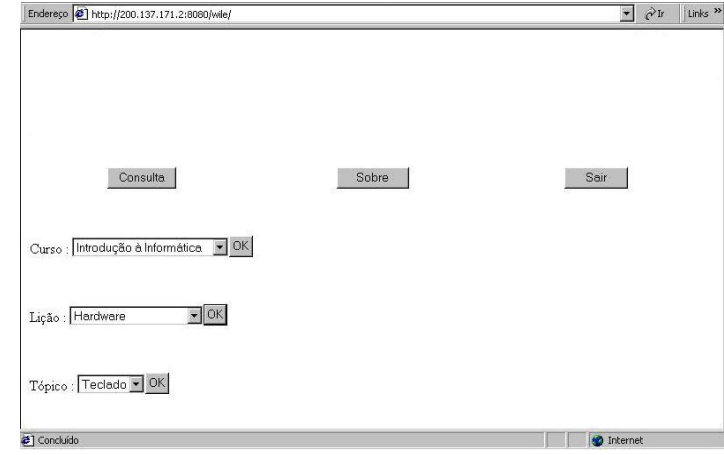

Figura 5: Consulta tópicos da lição

Escolhido o tópico da lição, o usuário deve clicar no botão Consulta, para executar a consulta nos bancos de dados locais cooperantes. O resultado da consulta 
pode ser uma página informando que o sistema não encontrou bancos de dados locais cooperantes conectados no momento ou não encontrou conteúdo relacionado ao tópico escolhido e, pode ser uma página com o conteúdo armazenado nos bancos de dados locais cooperantes conectados no momento, referentes ao tópico selecionado para consulta, conforme a figura 6.

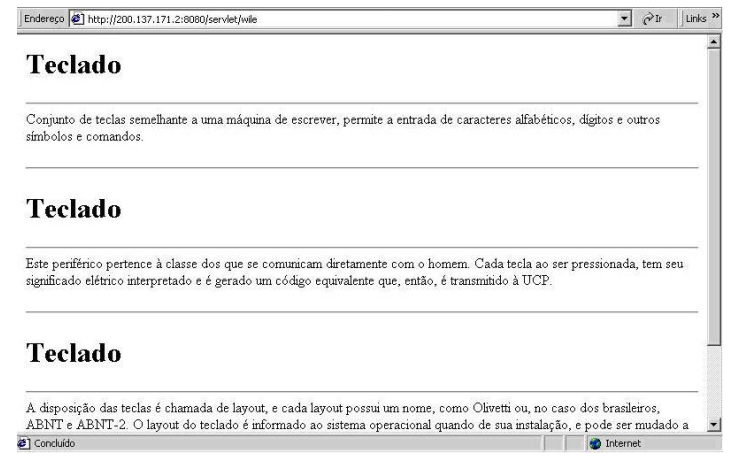

Figura 6: Resultado da Consulta Bem-Sucedida

\section{Conclusões}

O uso de bancos de dados locais em um ambiente colaborativo de aprendizagem baseado na WWW tem por objetivo atender aos requisitos de colaboração entre os estudantes participantes do ambiente. Dessa forma, a partir do ambiente, é possível realizar a disponibilização e a integração dos bancos de dados locais e cooperantes.

Tal integração permite que vários bancos de dados sejam vistos pelos estudantes como um único banco de dados, proporcionando a troca de informações entre os estudantes e a melhoria do processo de aprendizagem, onde a construção e o acúmulo de informação é feito através das bases do domínio do próprio ambiente, como também, através dos bancos de dados individuais de cada aluno

Além de proporcionar ao aluno uma aprendizagem ativa e colaborativa, o ambiente permite ao professor realizar um upload dos dados dos bancos de dados locais cooperantes, caso entenda que, após uma consulta global, ele venha a encontrar algum material que possa ser adicionado à base do domínio, assim, o upload pode enriquecer ainda mais o material didático fornecido aos usuários do ambiente.

Vale ressaltar que a consulta aos bancos de dados locais é uma busca refinada, visto que, o conteúdo dos bancos de dados é totalmente condizente com o que se deseja pesquisar, diferentemente das pesquisas realizadas através de ferramentas de busca na Internet, onde são pesquisas textuais e muitas das vezes trazem resultados indesejáveis.

\section{Referências bibliográficas}

AULANET: Disponível em http://www.les.inf.puc-rio.br/aulanet/. Acesso em 08/09/2004.

BUSSE, S.; KUTSCHE, R. D.; LESER U.; WEBER H.. Federated Information Systems: concepts, terminology and architectures. Technical Report Nr. 99-9. TU Berlin, 1999.

COMPUTER SUPPORTED COLLABORATIVE LEARNING (CSCL), Disponível em $<$ http://www.edb.utexas.edu/csclstudent/Dhsiao/theories.html $>$. Acesso em 08/09/2004. 
E-PROINFO: Disponível em <http://www.eproinfo.mec.gov.br/>. Acesso em 08/09/2004.

GOMES, R. C. G. Educação a distância: uma alternativa para a formação de professores e demais profissionais na sociedade do conhecimento. Florianópolis, 2000. 169 f. Dissertação de Mestrado em Engenharia de Produção, Programa de Pós-Graduação em Engenharia de Produção, UFSC, 2000.

HARASIN, L. On-line education: a new domain. In: Mason, Robin and Kaye, Anthon (eds). Mindweave: communications, computers and distance, 1989.

HILTZ, S.R.; TUROFF, M. Video plus virtual classroom for distance education: experience with graduate courses. Conference on Distance Education in DoD, 1993. National Defense University. Disponível em: $<$ http://eies.njit.edu/ turoff/Papers/dised2.htm> Acesso em 08/09/2004.

HTML (HyperText Markup Language): Disponível em <http://www.w3.org/MarkUp/>. Acesso em 08/09/2004.

JAVA: Disponível em <http://java.sun.com/>. Acesso em 08/09/2004.

JDBC (Java DataBase Conectivity): Disponível em $<$ http://java.sun.com/products/jdbc/>. Acesso em 08/09/2004.

ÖZSU, M. TAMER; VALDURIEZ, PATRICK. Princípios de Sistemas de Bancos de Dados Distribuídos. Editora Campus. 2001.

PORTER, L. Creating the virtual classroom. New York: John Wiley \& Sons, USA, 1997.

RMI (Remote Method Invocation): Disponível em $<$ http://java.sun.com/products/jdk/rmi/>. Acesso em 08/09/2004.

SERVLET: Disponível em <http://java.sun.com/products/servlet/>. Acesso em 08/09/2004.

TELEDUC: Disponível em <http://teleduc.nied.unicamp.br/>. Acesso em 08/09/2004.

THOMAS, G. et all, Heterogeneous Distributed Database System for Production Use, ACM Computing Surveys, v.22(3), Set, 1990

TINTO, V., GOODSELL, A., RUSSO, P. Building learning communities for new students: A summary of research findings of the Collaborative Learning Project. National Center on Postsecondary Teaching, Learning, and Assessment. 1995.

TODOROV, J. C. A importância da educação a distância. In: Educação a distância, v. 3, n. 4 e 5. p 5-6, dezembro/1993 - abril/1994, Brasília, INED.

VALENTE, J. A. O uso inteligente do computador na educação. Pátio, Ano 1, n. 1, p. 18-21, maio/julho de 1997.

WEBCT: Disponível em <http://www.webct.com/>. Acesso em 08/09/2004. 